\title{
Broadband, Temperature-Stable, Reflective Additives to Enhance Thermal Radiation Protection Systems
}

\section{Conference Paper}

\section{Author(s):}

Christidis, George (1); Koch, Ueli (1D; Poloni, Erik (1D; De Leo, Eva; Cheng, Bojun (1); Koepfli, Stefan M. (1); Dorodnyy, Alexander; Bouville, Florian (D); Fedoryshyn, Yuriy M.; Shklover, Valery; Leuthold, Juerg (i)

\section{Publication date:}

2020-07-14

\section{Permanent link:}

https://doi.org/10.3929/ethz-b-000456252

Rights / license:

In Copyright - Non-Commercial Use Permitted

Originally published in:

https://doi.org/10.1364/NOMA.2020.NoTu2C.4

\section{Funding acknowledgement:}

160184 - Design and manufacturing of heterogeneous photonic composites for aerospace applications (SNF) 


\title{
Broadband, Temperature-Stable, Reflective Additives to Enhance Thermal Radiation Protection Systems
}

\author{
George Christidis ${ }^{\text {a, }}$ Ueli Koch ${ }^{\text {a }}$, Erik Poloni ${ }^{\text {b}}$, Eva De Leo ${ }^{\text {a }}$, Bojun Cheng ${ }^{\text {a, }}$ Stefan M. Koepfli ${ }^{\text {a }}$, Alexander \\ Dorodnyy $^{\text {a }}$, Florian Bouville ${ }^{b}$, Yuriy Fedoryshyn ${ }^{\text {a }}$, Valery Shklover ${ }^{\text {a }}$, Juerg Leuthold a \\ ${ }^{a}$ Department of Information Technology and Electrical Engineering, ETH Zurich, 8092 Zurich, Switzerland \\ ${ }^{\mathrm{b}}$ Department of Materials, ETH Zurich, 8093 Zurich, Switzerland \\ Authore-mail address: george.christidis@ief.ee.ethz.ch
}

\begin{abstract}
Metal-dielectric platelets are introduced as additives to heat protection systems. The platelets feature high reflectivity across $700 \mathrm{~nm}$ and thermal stability up to $1000^{\circ} \mathrm{C}$. Impregnating aerospace heat shields improves thermal reflectivity by a factor 11 . $\odot 2020$ The Author(s)
\end{abstract}

\section{Introduction}

Thermal protection systems (TPS) are used in space missions that involve entry into a planet's atmosphere with hypersonic velocity [1]. The purpose of such a system is to protect the spacecraft against thermal loads experienced under these extreme conditions. The two main mechanisms of heat transfer in space re-entry conditions are convection due to the hypersonic hot gas flow around the spacecraft, and radiation resulting from ionization of atmospheric gases [2]. The generated radiation covers a broad electromagnetic spectrum that depends on the density and composition of atmospheric gases [3].

Current TPS technology deals only with the convective part of the heat flux, which is dominant for Earth re-entry. Yet, it is expected that future, manned missions in other planets will experience increased thermal radiation, which causes a significant contribution to the total heat flux [4]. One way to address thermal radiation is to introduce photonic additives inside conventional TPS for spacecrafts, Fig. 1(a), which allows for protection against convective heating and simultaneously reflects the radiation in the desired wavelength spectrum. These photonic inclusions need to be compatible with currently used technologies and display a good photonic response even at elevated temperatures [5].

In this work, we propose a new photonic heterostructure that combines the omnidirectional, broadband reflectivity of a metal (Ta) in the infrared with a stack of dielectric coatings $\left(\mathrm{SiO}_{2}\right.$ and $\left.\mathrm{Si}_{3} \mathrm{~N}_{4}\right)$, see Fig. 1(b). The dielectric coating is designed to provide thermal stability and extend the metal's photonic response towards the visible wavelengths. The simple (11-layer), heterostructure reflector is highly reflective $(\langle R\rangle=88.8 \%)$ across most of the wavelength range $(700-1420 \mathrm{~nm})$ relevant for Earth re-entry. Additionally, it exhibits stable and reversible photonic properties up to $1000{ }^{\circ} \mathrm{C}$, when thermally cycled. To evaluate its performance, doubled-sided, heterostructure platelets have been fabricated and impregnated in a composite matrix (resin). A reflectivity enhancement by a factor 11 was observed [6].
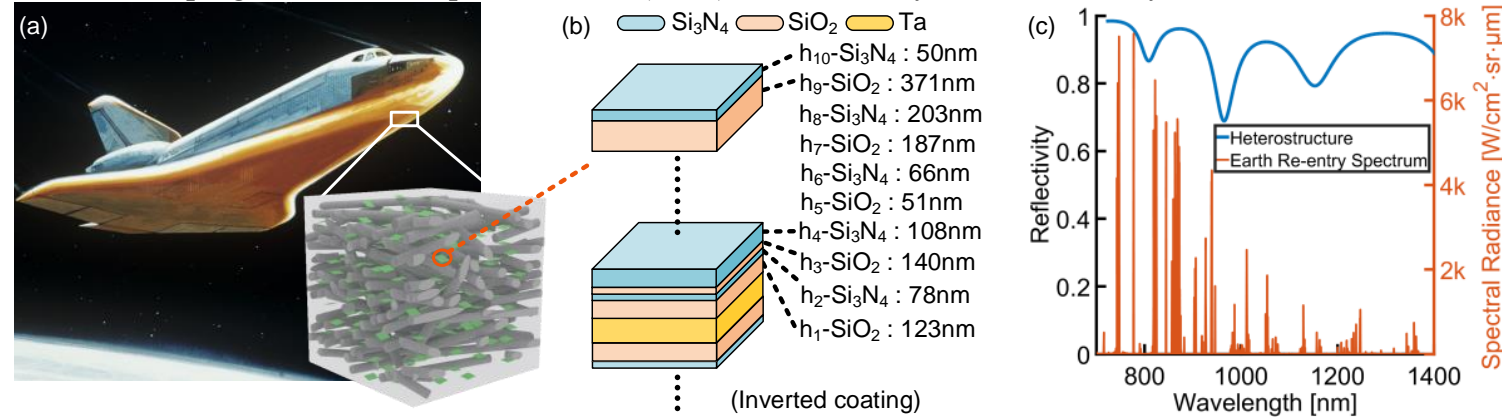

Fig. 1. (a) Photonically enhanced thermal protection system (TPS) used during planetary entry of a spacecraft. (b) Designed photonic heterostructure platelets composed of a metal (Ta) and dielectric coating $\left(\mathrm{SiO}_{2}\right.$ and $\left.\mathrm{Si}_{3} \mathrm{~N}_{4}\right)$. The structure has been optimized for high reflectivity and broadband performance from the visible to the near IR. (c) Simulated reflectivity of the photonic heterostructure (blue line) and relevant spectral radiance under Earth re-entry conditions (red line) [3].

\section{Design and Fabrication}

The goal is to design a material composite with a high average specular reflectivity $\langle R\rangle$ over a broad wavelength range $(700-1400 \mathrm{~nm})$ and to suppress resonant reflectivity features, Fig. 1(c). In order to achieve it, we combine the broadband reflectivity of a metal in the infrared with the reflective properties of a few dielectric layers. For this purpose, a dielectric coating has been designed by means of an evolution strategy (ES) algorithm [5] with the individual layer thicknesses as independent parameters, Fig. 1(b). Additionally, the composite needs to be stable under elevated temperatures. To meet the temperature stability requirements $\mathrm{Ta}, \mathrm{SiO}_{2}$ and $\mathrm{Si}_{3} \mathrm{~N}_{4}$ have been selected. The coating has then been fabricated using plasma enhanced chemical vapor deposition (PECVD) to deposit the $\mathrm{SiO}_{2}$ and 
$\mathrm{Si}_{3} \mathrm{~N}_{4}$ layers, while Ta has been deposited using sputtering. The double-sided photonic additives are separated from the supporting Si substrate by chemical etching. The collected additives are then mixed with an organic matrix (resin), and dropcasted to form the final sample. The platelet-impregnated organic matrix is shown in Fig. 2(b). The concentration of the platelets is high on the top side of the sample (red color) due to sedimentation.

\section{Results and Discussion}

The reflectivity and temperature stability of the photonic heterostructure (on substrate) has been characterized using a heating stage (Linkam 1500TS) connected to a Fourier transform infrared spectrometer (FTIR, Bruker v80). The coating has been thermally cycled between room temperature $\left(27^{\circ} \mathrm{C}\right)$ and $1000^{\circ} \mathrm{C}$ five times, Fig. 2(a). The measured reflectivity at $27^{\circ} \mathrm{C}$ shows good agreement for successive thermal cycles and the coating displays no degradation or material's phase changes when characterized by SEM and XRD. The small variance between different thermal cycling curves $\left(27^{\circ} \mathrm{C}\right)$ has been attributed to the thermal stability of the tungsten-halogen lamp, used for the sample's illumination. This is in agreement with the measured reflectivity curves at $1000^{\circ} \mathrm{C}$, which are identical for all cycles. The heterostructure's measured reflectivity (Fig. 2(c), blue curve) is similar in form and values to the simulated one, see Fig. 1(c), while the reflector retains its broadband, optical properties at high temperatures, Fig. 2(a).

Double-sided heterostructure platelets have been prepared as photonic additives for impregnation into a composite matrix (organic resin). The diffused reflectivity of the sample has been measured using a fiber coupled broadband source (tungsten lamp), a spectrometer and an integration sphere (Ocean Optics). Fig. 2(c) shows the photonic enhancement achieved by inserting these platelets into an inherent non-reflective material (resin). The low reflectivity of the resin $(\langle R\rangle=4.9 \%$, red curve) has been increased by a factor 11 to an average diffuse reflectivity of $55.1 \%$ (blue curve). This photonic enhancement has been achieved with no additional control of the additives' alignment in the matrix and is the result of the platelets' high omnidirectional, reflective properties.
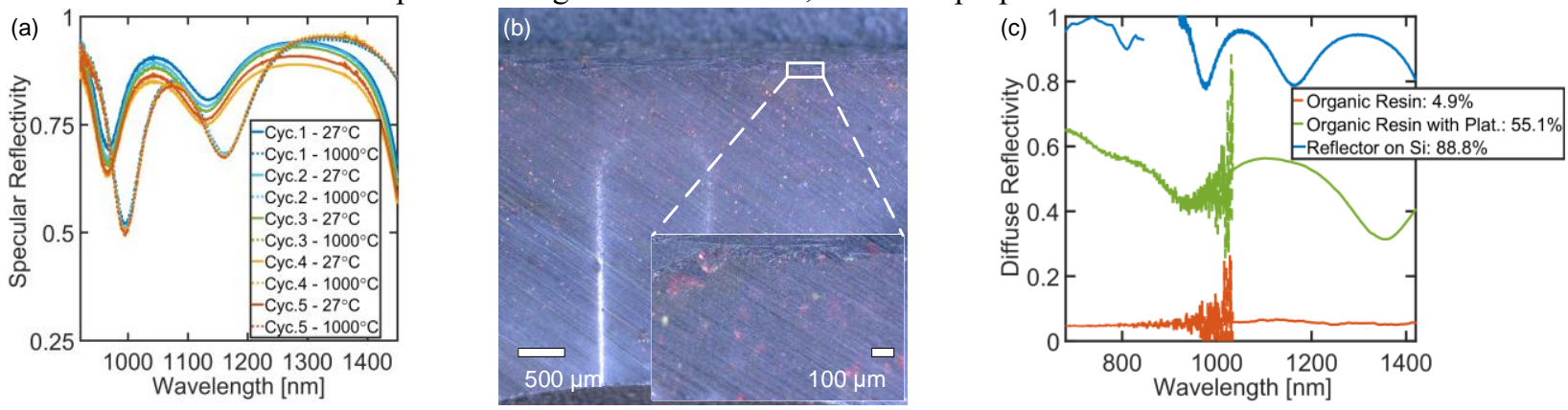

Fig. 2. (a) Reflectivity as a function of wavelength for different thermal cycles up to $1000^{\circ} \mathrm{C}$. The photonic heterostructure recovers its room temperature reflectivity values without showing any signs of degradation. (b) Light microscopy cross section of the composite matrix impregnated with photonic heterostructure platelets. The random alignment of platelets can be seen in the inset. (c) Diffuse reflectivity for pure organic resin matrix (red line), an organic resin matrix impregnated with heterostructure platelets (green line) and the heterostructure reflector on silicon (blue line) for comparison. A reflectivity enhancement from $4.9 \%$ to $55.1 \%$ is observed.

\section{Conclusion}

A simple, broadband and high-temperature stable heterostructure reflector has been designed and fabricated for enhancing thermal radiation protection systems such as heat shields used in Earth re-entry. The reflector shows hightemperature stability, when thermally cycled, broadband and high average reflectivity and is used as additives that are impregnated in non-photonic composite matrices to enhance their optical performance.

\section{Funding}

This work has been supported by the Swiss National Science Foundation (project: 200021-160184) and has been partially carried out in the Binnig and Rohrer Nanotechnology Center (BRNC).

\section{References}

[1] J. T. Howe, M. J. Green, and K. C. Weston, "Thermal shielding by subliming volume reflectors in convective and intense radiative environments," AIAA Journal 11, 989-994 (1973).

[2] M. Stackpoole, S. Sepka, I. Cozmuta, and D. Kontinos, "Post-Flight Evaluation of Stardust Sample Return Capsule Forebody Heatshield Material," in 46th AIAA Aerospace Sciences Meeting and Exhibit,. (American Institute of Aeronautics and Astronautics, 2008).

[3] A. M. Brandis, C. O. Johnston, B. A. Cruden, and D. K. Prabhu, "Equilibrium Radiative Heating from 9.5 to $15.5 \mathrm{~km} / \mathrm{s}$ for Earth Atmospheric Entry," Journal of Thermophysics and Heat Transfer 31, 178-192 (2017).

[4] J. H. Grinstead, M. J. Wright, D. W. Bogdanoff, and G. A. Allen, "Shock Radiation Measurements for Mars Aerocapture Radiative Heating Analysis," Journal of Thermophysics and Heat Transfer 23, 249-255 (2009).

[5] N. Komarevskiy, V. Shklover, L. Braginsky, C. Hafner, O. Fabrichnaya, S. White, and J. Lawson, "Design of Reflective, Photonic Shields for Atmospheric Reentry," Journal of Electromagnetic Analysis and Applications 3, 228-237 (2011).

[6] G. Christidis, U. Koch, E. Poloni, E. D. Leo, B. Cheng, S. M. Koepfli, A. Dorodnyy, F. Bouville, Y. Fedoryshyn, V. Shklover, and J. Leuthold, "Broadband, High-Temperature Stable Reflector for Aerospace Thermal Radiation Protection," ACS Applied Materials \& Interfaces 12, $9925-9934$ (2020). 